Check for updates

Cite this: RSC Adv., 2019, 9, 11281

\title{
Solvation effect and binding of rhaponticin with iron: a spectroscopic and DFT/TDDFT study $\dagger$
}

\begin{abstract}
Zhen Liu, (D) * Ling Xue, Yuan Jia, Boyu Lou and Juxiang Yang
In this article, both experimental and computational methods are employed to investigate the photophysics of rhaponticin (RH). The bathochromic shift was observed in absorption and fluorescence spectra with increasing solvent polarity, which implied that the charge transition of $\mathrm{RH}$ involved was $\pi \rightarrow \pi^{*}$. The results showed that $\mathrm{RH}$ possess strong intramolecular charge transfer (ICT), and the most important parameter to characterize the photophysical behavior of $\mathrm{RH}$ is the intermolecular hydrogen bonding ability of the solvent. The hydrogen bonding effect occurred at the localized electron-acceptor oxygen at the glycoside bond. Density functional theory (DFT) and time dependent density functional theory (TDDFT) were used to obtain the most stable structure, electronic excitation energy, dipole moments and charge distribution. The result was found to be 2.23 and $3.67 \mathrm{D}$ in ground state and excited state respectively. Fluorescence quenching of $\mathrm{RH}$ owing to the photoinduced electron transfer (PET) is facilitated in alkaline media. The $\mathrm{p} K_{\mathrm{a}}$ value of $\mathrm{RH}$ was 6.39, which defined $\mathrm{RH}$ as a highly efficient "off-on" switcher. The effect of different metal ions on the fluorescence spectra of $\mathrm{RH}$ was also investigated, and the fluorescence quenching of $\mathrm{RH}$ depended on the nature of ions. The best performance was accomplished for binding with the $\mathrm{Fe}^{3+}$ ion. The interactions of $\mathrm{RH}$ with the $\mathrm{Fe}^{3+}$ ion were studied by FT-IR and HPLC, and the binding parameter was calculated by the Stern-Volmer equation. The results obtained reveal the binding activity of $\mathrm{RH}$ can make this a candidate as a good source of new agents for thalassemic patients.
\end{abstract}

Received 11th December 2018 Accepted 12th March 2019

DOI: 10.1039/c8ra10153a

rsc.li/rsc-advances
Moreover, the hydroxyl groups at C-11 and methoxy group at C12 of act electron donor and the glycoside fragment functions as a receptor unit, and there exists electronic transition as $\pi \rightarrow \pi^{*}$, belong to polyphenol derivatives. Therefore, $\mathrm{RH}$ would show<smiles>COc1ccc(/C=C/c2cc(O)cc(O[C@@H]3OC(CO)[C@@H](O)[C@H](O)[C@H]3O)c2)cc1O</smiles>

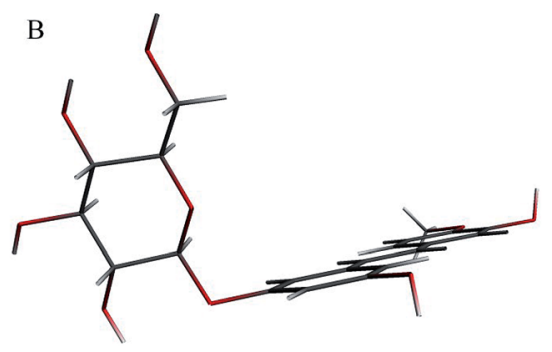

Fig. 1 (A): Chemical structure of rhaponticin (RH), (B): minimumenergy B3LYP/DZP structure of $\mathrm{RH}$.
School of Chemical Engineering, Xi'an University, No. 1 Keji 6th Road, Xi'an, Shaanxi 710065, China.E-mail: liuz@xawl.edu.cn

$\dagger$ Electronic supplementary information (ESI) available. See DOI: 10.1039/c8ra10153a
The most serious complication of $\beta$-thalassemia is iron overload, and it is vital to control it within a reasonable range. thalassemia has been presented in a paper by Crisponia and Remelli. ${ }^{1}$ Design and development of iron selective fluorescent chemosensors has caused great concern, due to their importance in many biological and environmental processes.

As a major representative of the stilbene glucoside compounds, rhaponticin (RH, Fig. 1) exists widely in medicinal plant of Rheum L., such as Rheum officinale, Rheum undulatum, Rheum hotaoense and Rheum palmatum. ${ }^{2}$ Previous works of our working-team showed that $\mathrm{RH}$ possessed various biological Moreover, the atoms of the stilbene moiety are nearly coplanar in the molecule of $\mathrm{RH}$, and the dihedral angle between ring (C2$\mathrm{C} 7)$ and ring (C10-C15) is $9.0^{\circ}$. The hydrogen bonds exist among the hydroxyl group $(-\mathrm{OH})$, methoxy group $\left(-\mathrm{OCH}_{3}\right)$ and $\mathrm{O}$ atom, and link the molecules into a complicated $3 \mathrm{D}$ framework. ${ }^{3}$ 
optical property which is highly sensitive to its surroundings, it is reasonable to use it as a polarity probe and it also can be applied in medicine and chemistry. ${ }^{6}$ The multiple pharmacological activities make it be worth carrying out a further study on the photophysical property of $\mathrm{RH}$.

Solvent effect is closely related to the nature and extent of solute-solvent interactions developed locally in the immediate vicinity of solute. One key approach to understand solvent effects is the solvent-induced changes in the electronic transition of solutes generally referred to as solvatochromism. The application of computational methods to studies in photophysics chemistry is instrumental in understanding phosphorescent mechanisms. ${ }^{7}$ In recent years, approaches using density functional theory (DFT) and time dependent density functional theory (TDDFT) have received large acceptance for describing the ground state and excited state properties of photoluminescence molecules, such as geometrical parameters, energy gap, dipole moments and excited energy. ${ }^{8}$

Until now, there is no report available in the literature on the luminescent mechanism of $\mathrm{RH}$. Therefore, we focus on the photophysical properties of $\mathrm{RH}$, and we present a comparative study of the experimental absorption and fluorescence spectra with DFT/TDDFT calculations in this work. The binding of RH with iron was also studied by spectroscopic, chromatographic methods, which would have a great significance in pharmacology and clinical medicine as well as methodology.

\section{Materials and methods}

$\mathrm{RH}$ was isolated by authors from $R$. hotaoense, ${ }^{3}$ and its purity was evaluated to be above 99\% (HPLC and spectral analysis). The stock solutions of $\mathrm{RH}\left(1.5 \times 10^{-5} \mathrm{M}\right)$ were prepared by dissolving appropriate amount of $\mathrm{RH}$ in $10 \mathrm{~mL}$ different solvents, and the solvents applied were analytical grade and used without further purification.

All fluorescence spectra were carried out on an F-7000 fluorescence spectrometer (Hitachi, Japan) equipped with a $150 \mathrm{~W}$ xenon lamp, and the slit width was $5 \mathrm{~nm}$. All absorption spectra were recorded by UV-2501PC spectrophotometer (Shimadzu, Japan) in the range of 200-400 $\mathrm{nm}$. Fourier Transform Infrared Spectroscopy (FT-IR) spectra were obtained using an EQUINOX55 fourier transformed infrared spectrometry (Bruker
Company, Germany) in the range of 4000-600 $\mathrm{cm}^{-1}$. HPLC analyses were performed on an Agilent 1200 series system (Agilent, Santa Clara, CA), which consisted of an auto sampler, a Zorbax carbohydrate column $(0.46 \times 15.0 \mathrm{~cm})$, a solvent system of acetonitrile/methanol (30:70), at a flow rate of 1.0 $\mathrm{mL} \min ^{-1}$ with the UV detector wavelength set at $324 \mathrm{~nm}$, and the injection volume was $20 \mu \mathrm{L}$. Data were acquired and processed with an Agilent ChemStation software external calibration.

The solvatochromic method was used to calculate the dipole moment in ground and excited state. All calculations in the present study were performed by using the Amsterdam Density Functional package (ADF) 2010.01 program. ${ }^{9}$ Geometry optimization in the ground state was carried out using B3LYP density functional calculations, with the DZP basis sets. ${ }^{\mathbf{1 0}}$ The lowest singlet excited-states of both the isolated $\mathrm{RH}$ and the hydrogen-bonded RH were calculated using time-dependent density functional theory (TD-DFT) with B3LYP hybrid functional and the TZVP basis set. ${ }^{11}$

\section{Results and discussions}

\section{Solvent effects on the absorption and fluorescence spectra of RH}

Theoretical density functional theory (DFT) calculations were performed to gain more insight into the electronic properties of these systems that appear as promising molecules for lightharvesting applications. ${ }^{12}$ Fig. $1 \mathrm{~B}$ displays the minimumenergy molecular geometry computed for RH at the B3LYPDZP level. As expected, RH exhibits the typical boat conformation, and the atoms of the stilbene moiety are coplanar, suggesting that an efficient $\pi$-conjugation can operate between the donor and acceptor units in RH molecule. The absorption and fluorescence spectra of $\mathrm{RH}$ in different solvents were presented in Fig. 2. The values of the absorption and fluorescence spectral maxima were listed in Table 1 . As we know, the shift can be described as hypsocromic or bathochromic depending on whether the absorption maximum occurs at a shorter or longer wavelength respectively. As seen from Fig. 2A, the absorption maximum is obtained around $311 \mathrm{~nm}$ in dioxane and it is about $327 \mathrm{~nm}$ in water. In the present study, the absorption spectra shift to longer wavelength with increasing solvent polarity,
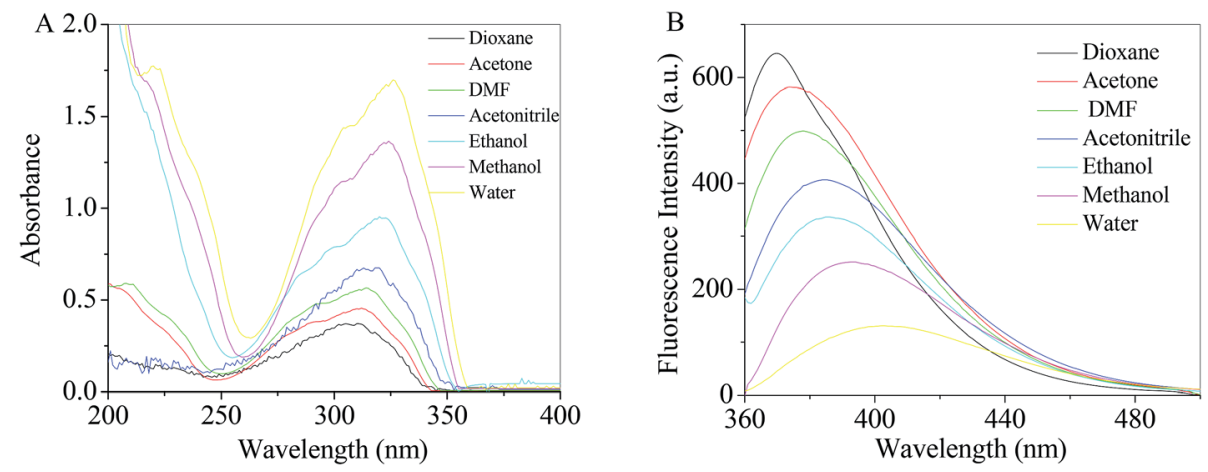

Fig. 2 Absorption (A) and fluorescence (B) spectra of $\mathrm{RH}$ in different solvents. 
Table 1 The physical or spectral parameters of solvents and spectral data of $\mathrm{RH}^{a}$

\begin{tabular}{|c|c|c|c|c|c|c|c|c|}
\hline Solvents & $\lambda_{\mathrm{a}}(\mathrm{nm})$ & $\lambda_{\mathrm{F}}(\mathrm{nm})$ & $\Delta \lambda(\mathrm{nm})$ & $I_{\mathrm{F}}$ (a.u.) & $\varepsilon$ & $n$ & $F 1$ & $F 2$ \\
\hline 2,4-Dioxane & 311 & 370 & 59 & 656 & 2.21 & 1.422 & 0.044 & 0.671 \\
\hline Toluene & 312 & 371 & 59 & 642 & 2.38 & 1.497 & 0.029 & 0.700 \\
\hline Diethylether & 312 & 372 & 60 & 637 & 4.27 & 1.353 & 0.371 & 0.851 \\
\hline Ethyl acetate & 312 & 372 & 60 & 628 & 6.02 & 1.370 & 0.493 & 1.001 \\
\hline Chloroform & 312 & 373 & 61 & 615 & 4.81 & 1.443 & 0.371 & 0.985 \\
\hline $\mathrm{THF}$ & 314 & 375 & 61 & 610 & 7.52 & 1.407 & 0.550 & 1.103 \\
\hline Acetone & 314 & 376 & 62 & 595 & 21.01 & 1.359 & 0.795 & 1.282 \\
\hline DMF & 314 & 378 & 64 & 501 & 36.70 & 1.431 & 0.844 & 1.424 \\
\hline DMSO & 316 & 381 & 65 & 456 & 47.20 & 1.479 & 0.843 & 1.496 \\
\hline Acetonitrile & 321 & 387 & 66 & 402 & 38.80 & 1.344 & 0.861 & 1.332 \\
\hline Butanol & 319 & 385 & 64 & 385 & 17.40 & 1.399 & 0.749 & 1.296 \\
\hline Propanol & 320 & 386 & 66 & 363 & 1.385 & 20.60 & 0.781 & 1.303 \\
\hline Ethanol & 323 & 390 & 67 & 343 & 24.55 & 1.361 & 0.812 & 0.651 \\
\hline Methanol & 325 & 394 & 69 & 252 & 33.70 & 1.329 & 0.860 & 0.648 \\
\hline Water & 327 & 403 & 76 & 109 & 78.50 & 1.333 & 0.914 & 0.683 \\
\hline
\end{tabular}

indicating RH exhibits a positive solvatochromism and $\pi \rightarrow \pi^{*}$ transition. According to Jayabharathi, the bathochromic shift happens when the dipole moment of the compound increases during the electronic transition i.e., the dipole moment of excited state is higher compared to that in the ground state $\left(\mu_{\mathrm{e}}>\right.$ $\mu_{\mathrm{g}}$ ) and the excited state is formed in solvent cage of already partly oriented solvent molecules. ${ }^{13}$ In such cases, the relaxed excited state $S_{1}$ will be energetically stabilized relative to the ground state $S_{0}$ and a significant red shift of the absorption will be observed.

At the same time, the fluorescence spectral studies of $\mathrm{RH}$ with excitation wavelength of $355 \mathrm{~nm}$ were carried out in different solvents (Fig. 2B), the peak maximum shifted from 370 to $403 \mathrm{~nm}$ (bathochromic shift) as the solvent polarity increased (dioxane to water). Meantime, the Stokes shift of RH in dioxane is $56 \mathrm{~nm}$, whereas the Stokes shifts in water increases to $76 \mathrm{~nm}$, respectively. The increasing Stokes shift in polar protic solvents suggests that the fluorescent state of RH is the intramolecular charge transfer (ICT) feature.

Table 1 and Fig. 2B show fluorescence quenching and redshift behavior of RH in protic solvents compared with that in aprotic solvent. On the one hand, in a polar protic solvent with a relatively high hydrophilicity (e.g., methanol or water), $\mathrm{RH}$ takes a stretched conformation with the plane of stilbene moiety. In a non-polar solvent with a high hydrophobicity (e.g., dioxane), the conformation of the RH molecule is twisted and the twisted intramolecular charge transfer (TICT) state and selfassociation fluorescence emission emerge. ${ }^{14}$ On the other hand, ICT state is more likely to relax by non-radiative processes than by radiative processes, which results in quenching of $\mathrm{RH}$ in protic solvents. Moreover, quenching and red-shift are mainly therefore clearly associated with hydrogen bonding, and the excitated-state hydrogen bond strengthening effect of RH with protic solvents..$^{15}$ When the acceptor (oxygen atom at glycoside band) is hydrogen-bonded, a more charge-separated chromophore is more stabilized and the hydrogen bond in the corresponding electronic excited state is strengthened. Therefore, the quenching and red-shift in proic solvent is caused by the TICT process and excited-state hydrogen bond strengthening effect. In addition, as shown in Table 1, the effect of solvents on the emission spectra of RH is more remarkable than that on absorption spectra (absorption and emission wavelength shifts 16 and $33 \mathrm{~nm}$ from dioxane to water, respectively), which implies that the less polar nature of RH in the ground state than in excited state and the ground state energy distribution is not affected by the solvents.

\section{Determination of dipole moments of RH}

The dipole moment of the ground and excited state is related to the electron distribution, which will be affected by various solvents. Thus, it is important to study the dipole moments of ground and exited state of $\mathrm{RH}$ for it offers information about the change in the electronic distribution on excitation. According to Valeur et al. ${ }^{16}$ quantum mechanical second-order perturbation theory of absorption $\left(\nu_{\mathrm{a}}\right)$ and fluorescence $\left(\nu_{\mathrm{f}}\right)$ band shifts in different solvents of varying permittivity $(\varepsilon)$ and refractive index (n) relative to the band position of a solute molecule is obtained. In order to avoid the hydrogen bonding between $\mathrm{RH}$ and protic solvents, we choose the aprotic solvents in Table 1, from 2,4dioxane to acetonitrile, and the related equations are as followed:

$$
\begin{gathered}
\nu_{\mathrm{a}}-\nu_{\mathrm{f}}=m_{1} f(\varepsilon, n)+\text { const } \\
\nu_{\mathrm{a}}+\nu_{\mathrm{f}}=-m_{2}[f(\varepsilon, n)+2 g(n)]+\mathrm{const}
\end{gathered}
$$

where

$$
f(\varepsilon, n)=\frac{2 n^{2}+1}{n^{2}+2}\left[\frac{\varepsilon-1}{\varepsilon+2}-\frac{n^{2}-1}{n^{2}+2}\right]
$$

with

$$
g(n)=\frac{3}{2}\left[\frac{\left(n^{4}-1\right)}{\left(n^{2}+2\right)^{2}}\right]
$$


where, $\varepsilon$ is the dielectric constant and $n$ is the refractive index.

$$
m_{1}=\frac{2\left(\mu_{\mathrm{e}}-\mu_{\mathrm{g}}\right)^{2}}{h c a^{3}}
$$

and

$$
m_{2}=\frac{2\left(\mu_{\mathrm{e}}^{2}-\mu_{\mathrm{g}}^{2}\right)}{h c a^{3}}
$$

where, $h$ is the Planck's constant and $c$ is the velocity of light in vacuum, and $m_{1}$ and $m_{2}$ were calculated from the eqn (1) and (2) denoting the absorption and fluorescence band shifts. The values of ground state and excited state dipole moment, $\mu_{\mathrm{g}}$ and $\mu_{\mathrm{e}}$, were obtained from eqn (5) and (6): ${ }^{17,18}$

$$
\begin{aligned}
& \mu_{\mathrm{g}}=\frac{m_{2}-m_{1}}{2}\left(\frac{h c a^{3}}{2 m_{1}}\right)^{\frac{1}{2}} \\
& \mu_{\mathrm{e}}=\frac{m_{2}+m_{1}}{2}\left(\frac{h c a^{3}}{2 m_{1}}\right)^{\frac{1}{2}}
\end{aligned}
$$

and

$$
\mu_{\mathrm{e}}=\frac{m_{1}+m_{2}}{m_{2}-m_{1}} \mu_{\mathrm{g}}\left(m_{2}>m_{1}\right)
$$

The value of the solute cavity radius $a$ was calculated from the molecular volume by Suppan's equation: ${ }^{19}$

$$
a=\left(\frac{3 M}{4 \pi \delta N}\right)^{\frac{1}{3}}
$$

Here $N$ is the Avogadro's number, $M$ is the solute's weight and $\delta$ is the solid-state density of solute molecule. Here $m$ is the slope of the linear plot of permittivity $(\varepsilon)$ and refractive index $(n)$ versus Stokes shift (Fig. S1A and B, ESI $\dagger$ ). Then the dipole moment can be calculated by above parameters. One can see that $\mu_{\mathrm{g}}=2.23 \mathrm{D}$ and $\mu_{\mathrm{e}}=3.67 \mathrm{D}$. The dipole moment of the $\mathrm{RH}$ in excited state is higher than that in ground state owing to the change in electronic distribution. This result means a more polar and stable structure in excited state than that of in ground state, which is consistent with the result of absorption spectra.

\section{Theoretical calculation using DFT and TDDFT}

Fig. 3 shows the orbital energy levels of the highest occupied molecular orbital (HOMO) and the lowest unoccupied molecular orbital (LUMO) and their energy gap for RH in dioxane, acetone, ethanol and water. And the calculated energy gaps, wavelengths of the lowest electronic transitions, oscillator strengths, and main orbital transition contribution and corresponding CI coefficients of the low-lying electronically excited states of RH in four solvents were listed in Table 2. The observed positive solvatochromism is reproduced by DFT/TDDFT calculations, and the maximum oscillator strengths are corresponding to the HOMO $\rightarrow$ LUMO+2 orbital transition. The occupied orbital was mainly localized on the stilbene unit whereas the LUMO+2 is centered on the glycoside part, suggesting a strong propensity for intramolecular charge transfer from the electron-donor hydroxyl groups at C-11 and methoxy group at C-12 to the glycoside acceptor, and therefore correspond to $\pi \rightarrow \pi^{*}$ transitions. As seen from Fig. 3 that the energy

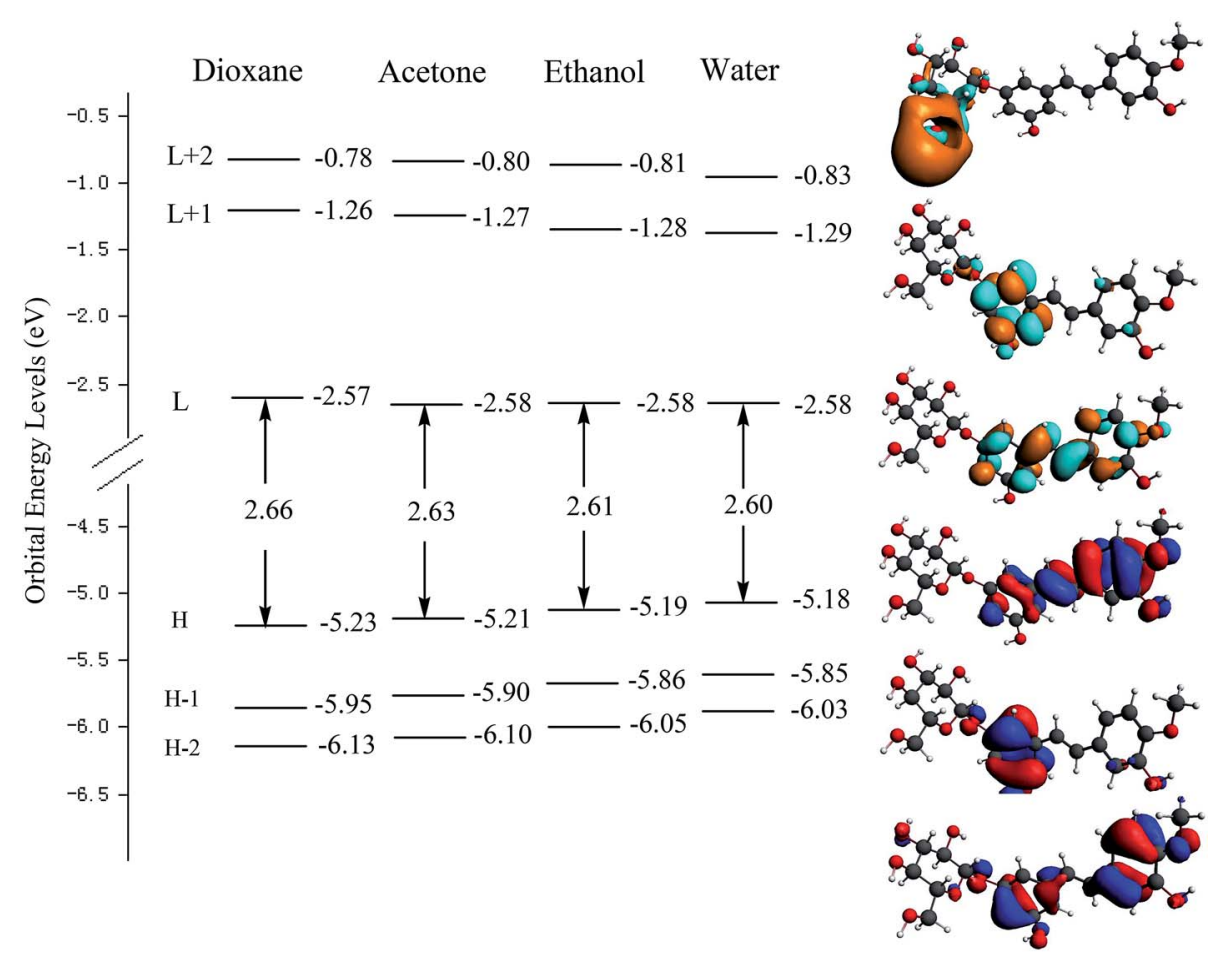

Fig. 3 Electron density contours and orbital energies calculated for the HOMOs and LUMOs of RH at the B3LYP/DZP level. H and L denote HOMO and LUMO, respectively. 
Table 2 Calculated energy gap $\left(E_{g}\right)$, oscillator strength $(f)$ and main orbital transition contribution (Contrib.) and absorption wavelength for RH in different solvents

\begin{tabular}{|c|c|c|c|c|c|c|}
\hline Solvent & Contrib. & CI component ${ }^{a}$ & Theory (nm) & Experiment (nm) & $E_{\mathrm{g}}(\mathrm{eV})$ & $f$ \\
\hline Dioxane & $\mathrm{HOMO} \rightarrow \mathrm{LUMO}+2$ & 0.951 & 315 & 311 & 2.66 & 0.243 \\
\hline Toluene & $\mathrm{HOMO} \rightarrow \mathrm{LUMO}+2$ & 0.904 & 320 & 314 & 2.63 & 0.246 \\
\hline Water & $\mathrm{HOMO} \rightarrow \mathrm{LUMO}+2$ & 0.943 & 339 & 327 & 2.60 & 0.252 \\
\hline
\end{tabular}

${ }^{a}$ The transition was shown by an arrow from one orbital to another, followed by its percentage CI (configuration interaction) component.

level of the HOMO increases from $-5.23 \mathrm{eV}$ in dioxane to $-5.18 \mathrm{eV}$ in water, suggesting the intermolecular hydrogen bonding raises the energy level of the HOMO. Meantime, the energy level of the LUMO decreases slightly from -2.57 to $-2.58 \mathrm{eV}$ from dioxane to water, indicating that intermolecular hydrogen bonding shows less effect to the electronically excited state of $\mathrm{RH}$ than ground state. ${ }^{20}$ The energy gap $\left(E_{\mathrm{g}}\right)$ between the HOMO and LUMO reduces from 2.66 to $2.60 \mathrm{eV}$ from dioxane to water. The reducing energy gap between the HOMOs and LUMOs is consistent with the absorption and fluorescence spectral red-shift of $\mathrm{RH}$ in polar solvents. On the other hand, from dioxane to water, there is a quasi parallel energy stabilization of the HOMO-2 and HOMO and a concomitant small destabilization of the LUMO. These evolutions result in the decrease of the HOMO $\rightarrow$ LUMO and HOMO $\rightarrow($ LUMO +2$)$ gap and thus in a bathochromic shift of the corresponding spectral behavior. $^{21}$

The electronic excitation energies and corresponding oscillator strengths of RH and the hydrogen-bonded $\mathrm{RH}$ are calculated using the TDDFT method and presented in Table 3. For both the isolated $\mathrm{RH}$ and the hydrogen-bonded $\mathrm{RH}$, the $\mathrm{S}_{1}$ state has the largest oscillator strength, which means that both of them can be initially photoexcited to the $S_{1}$ state. The excitation energy of the $S_{1}$ state is calculated to be at 4.11 and $3.92 \mathrm{eV}$ for $\mathrm{RH}$ and the hydrogen bonded $\mathrm{RH}$, suggesting that the hydrogen bonding induces a decrease for the excitation energy of the $S_{1}$ state, and the hydrogen bond is strengthened in this excited state..$^{22,23}$

The orbital transition contributions for the lowest single states $\mathrm{S}_{1}$ state of the isolated $\mathrm{RH}$ and the hydrogen-bonded $\mathrm{RH}$ are also presented in Table 3 . It can be seen that only the

Table 3 Calculated TD-DFT excitation energy $(E)$ and corresponding oscillator strengths (in the parentheses) of $\mathrm{RH}$ and the hydrogenbonded RH complex at the TD-DFT/B3LYP/TZVP level. The orbital transitions $\left(\mathrm{S}_{0} \rightarrow \mathrm{S}_{1}\right)$ and contributions are also listed. $\mathrm{HOMO}: \mathrm{H}$, LUMO: L

\begin{tabular}{lll}
\hline & RH & $\begin{array}{l}\text { Hydrogen-bonded } \\
\text { RH complex }\end{array}$ \\
\hline $\mathrm{S}_{1}$ & $4.11(0.241)$ & $3.92(0.261)$ \\
$\mathrm{S}_{2}$ & $\mathrm{H} \rightarrow \mathrm{L}(93.2 \%)$ & $\mathrm{H} \rightarrow \mathrm{L}(94.3 \%)$ \\
$\mathrm{S}_{3}$ & $4.30(0.034)$ & $4.25(0.123)$ \\
$\mathrm{S}_{4}$ & $4.52(0.202)$ & $4.48(0.082)$ \\
$\mathrm{S}_{5}$ & $4.68(0.023)$ & $4.62(0.023)$ \\
$\mathrm{S}_{6}$ & $4.85(0.253)$ & $4.78(0.232)$ \\
& $4.92(0.021)$ & $4.86(0.051)$
\end{tabular}

HOMO and LUMO are involved in the $\mathrm{S}_{1}$ state. To depict the nature of the lowest singlet excited state $S_{1}$, the HOMO and LUMO of both the RH and the hydrogen-bonded $\mathrm{RH}$ have been calculated and are shown in Fig. 4. As shown in Fig. 4, the electron density on the hydroxyl oxygen atom at C-11 in hydrogen-bonded $\mathrm{RH}$ is also remarkably decreased after the HOMO-LUMO transition. However, due to the formation of the intermolecular hydrogen bonds, the intramolecular hydrogen bond between the hydroxyl groups at C-11 and methoxy group at C-12 is not strengthened. On the contrary, the intermolecular hydrogen bond is significantly strengthened due to the remarkable decrease of the electron density on the hydroxyl oxygen atom at $\mathrm{C}-11 .^{24}$ Moreover, compared to $\mathrm{RH}$, the calculated absorption of hydrogen-bonded RH shift to longer wavelengths ( $c a .5 \mathrm{~nm}$, Fig. S2 $\dagger$ ). Compared with $\mathrm{RH}$, the formation of the intermolecular hydrogen bonds will stabilize both the HOMO and the LUMO of the hydrogenbonded form. The HOMO-LUMO energy decreases from $2.55 \mathrm{eV}$ in the RH form to $2.36 \mathrm{eV}$ in the hydrogen-bonded form respectively, which should be responsible for the $5 \mathrm{~nm}$ red shift of the $S_{1}$ absorption for the hydrogen bonded RH. In addition, it can be found that the HOMO and LUMO are the $\pi$ and $\pi^{*}$ character for both the $\mathrm{RH}$ and hydrogen bonded $\mathrm{RH}$, suggesting that the $S_{1}$ state is of the $\pi-\pi *$ feature.

Moreover, the value of the dipole moments $\left(D_{\mathrm{m}}\right)$ in the excited states is $3.87 \mathrm{D}$ (Table 4), and the difference between the dipole moments of the excited and ground states is $1.31 \mathrm{D}$, indicating that the value of dipole moment in the excited state of $\mathrm{RH}$ is lager, which explains well the experimental observations that RH shows a bathochromic shift of spectra and the

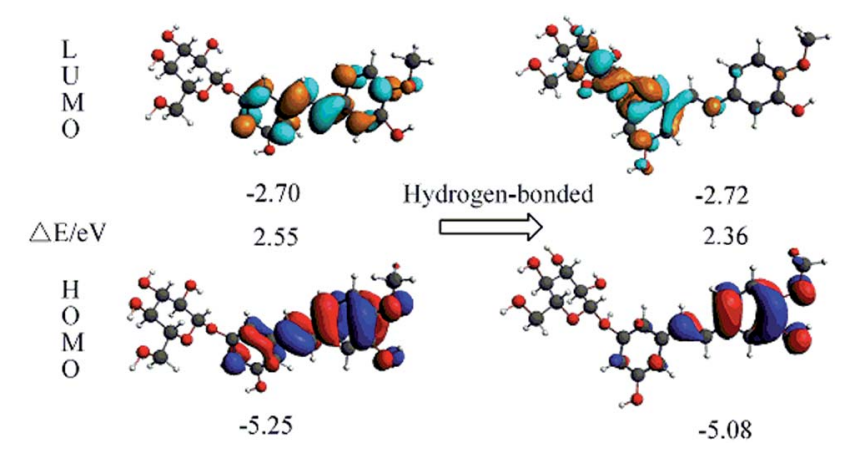

Fig. 4 Calculated for the HOMOs and LUMOs of RH (left) and the hydrogen-bonded $\mathrm{RH}$ with the oxygen atom at glycosidic bond (right). The orbital energies and energy gaps are also shown. 
Table 4 Calculated dipole moment (debye) for the ground $\left(\mu_{\mathrm{g}}\right)$ and the excited $\left(\mu_{\mathrm{e}}\right)$ states of $\mathrm{RH}$ and hydrogen bonded $\mathrm{RH}$ in vacuum ${ }^{a}$

\begin{tabular}{|c|c|c|c|c|c|c|c|}
\hline \multirow[b]{2}{*}{ Compound } & \multicolumn{3}{|c|}{ Experimental } & \multicolumn{3}{|c|}{ Theoretical } & \multirow{2}{*}{$\frac{\text { Calculation }}{\lambda_{\mathrm{a}}(\mathrm{nm})}$} \\
\hline & $\mu_{\mathrm{g}}$ & $\mu_{\mathrm{e}}$ & $\Delta \mu$ & $\mu_{\mathrm{g}}$ & $\mu_{\mathrm{e}}$ & $\Delta \mu$ & \\
\hline RH & 2.23 & 3.67 & 1.44 & 2.56 & 3.87 & 1.31 & 324 \\
\hline $\mathrm{RH} \cdots \mathrm{H}$ & - & - & - & 6.82 & 10.58 & 3.76 & 329 \\
\hline
\end{tabular}

large Stokes shift with increasing solvent polarity. The results of theoretical calculations have previously been shown to be in good agreement with experimental data.

\section{Effect of $\mathrm{pH}$ on the fluorescence spectra of $\mathrm{RH}$}

The $\mathrm{pH}$ effects on the emission and excitation spectra of $\mathrm{RH}$ were investigate in methanol. As seen from Fig. 5A, when the excitation wavelength is set at $340 \mathrm{~nm}, \mathrm{RH}$ exhibits a maximum around $390 \mathrm{~nm}$. The intensity decreases gradually with increasing $\mathrm{pH}$ along with a red shift (from 390 to $402 \mathrm{~nm}$ ). Fluorescence excitation spectra of $\mathrm{RH}$ are the similar response to the change of $\mathrm{pH}$ (Fig. 5B). After careful titration from $\mathrm{pH} 3$ to 11 , the emission intensity decreases practically eleven times (fluorescence quenching, $\mathrm{FQ}=11.43$ ) (Fig. $5 \mathrm{~B}$ insert). The $\mathrm{p} K_{\mathrm{a}}$ value of $\mathrm{RH}$ is calculated to be 6.39 by the eqn (11). ${ }^{25}$

$$
\log \left[\left(I_{\mathrm{F} \max }-I_{\mathrm{F}} / I_{\mathrm{F}}-I_{\mathrm{F} \min }\right)\right]=\mathrm{pH}-\mathrm{p} K_{\mathrm{a}}
$$

The $\mathrm{p} K_{\mathrm{a}}$ result suggests that $\mathrm{RH}$ is highly efficient "off-on" switchers for $\mathrm{pH}$, and also it is a potential useful sensor for higher $\mathrm{pH}$ measurement. Moreover, from these changes the emission is switched "off-on" between ca. pH 6.8 and 8.0. This switching process is also found to be reversible. These changes are of such magnitude that they can be considered as representing two different "states", where the fluorescence emission is "switched off" in alkaline solution and "switched on" in acidic solution. These changes are due to the deprotonation of the hydroxyl groups of $\mathrm{RH}$ at 4-C and 11-C, which engages in photoinduced electron transfer (PET) quenching of $\mathrm{RH}$ excited state and upon protonation of the hydroxyl groups, and the quenching process is substantially removed. ${ }^{26}$

\section{Effect of metal ions on the fluorescence spectra of RH}

The effects of different metal ions on the fluorescence spectra of $\mathrm{RH}$ are investigated. The presence of metal ions in RH solution as guests is signaled by fluorescence quenching (FQ). The FQ value is determined from the ratio of the maximum fluorescence intensity (solution free of metal ions) to the one after metal ions addition. Fig. 6 presents the FQ values for $\mathrm{RH}$ in the presence of different metal ions at the same concentration of $10.0 \mu \mathrm{M}$. As seen these values depends on the nature of the added ions and their ability to coordinate with the $\mathrm{RH}$ ligands. The highest FQ value is observed in the presence of $\mathrm{Fe}^{3+}$ ion. The binding ability of $\mathrm{RH}$ with different concentrations of $\mathrm{Fe}^{3+}$ ion is investigated by the spectroscopic and chromatographic methods.

As shown in Fig. 7A, the fluorescence intensity of $\mathrm{RH}$ drops sharply with increasing concentrations of $\mathrm{Fe}^{3+}$ ion along with the maxima bathochromic shift $9 \mathrm{~nm}$. The absorbance of $\mathrm{RH}$ increases and maxima shifts towards shorter wavelength region with the addition of $\mathrm{Fe}^{3+}$ ion. The spectral results indicate that $\mathrm{Fe}^{3+}$ ion binds with $\mathrm{RH}$ and a non-luminance $\mathrm{RH}-\mathrm{Fe}^{3+}$ complex forms.

In order to eliminate the influence of water and complex of $\mathrm{RH}-\mathrm{Fe}^{3+}$, FT-IR measurements were performed in the absence and presence of $\mathrm{Fe}^{3+}$ ion. The major differences in FT-IR spectra are observed in the regions of 1500, 1660 and $3300-3340 \mathrm{~cm}^{-1}$. A sharp peak near $1660 \mathrm{~cm}^{-1}$ appears after addition of $\mathrm{Fe}^{3+}$ ion, which causes by $\mathrm{H}_{2} \mathrm{O}$. The peak at $3309 \mathrm{~cm}^{-1}$ (Fig. 8A) related to the large amount of $-\mathrm{OH}$ in the $\mathrm{RH}$ molecule, and in the presence of $\mathrm{Fe}^{3+}$ ion, the hydroxyl peak shifts to $3336 \mathrm{~cm}^{-1}$. According to the result of FT-IR spectra, we can confirm that the formation of $\mathrm{RH}-\mathrm{Fe}^{3+}$ complex. The addition of $\mathrm{Fe}^{3+}$ ion results in noticeable decrease of transmittance of hydroxyl peak due to $\mathrm{H}$-bonded hydroxyls and the increasing intensity of the bridged $\mathrm{OH}$ groups. Double bond $\mathrm{C}=\mathrm{C}$ stretches at $1500 \mathrm{~cm}^{-1}$ and the decreasing intensity could be attributed to the contraction of the aromatic ring after the addition of $\mathrm{Fe}^{3+}$ ion. On the other hand, as seen from Fig. $8 \mathrm{~B}$ that the intensity and peak area decrease with the addition of $\mathrm{Fe}^{3+}$ ion, and the peak time changes slightly from 1.57 to $1.65 \mathrm{~min}$. The HPLC result
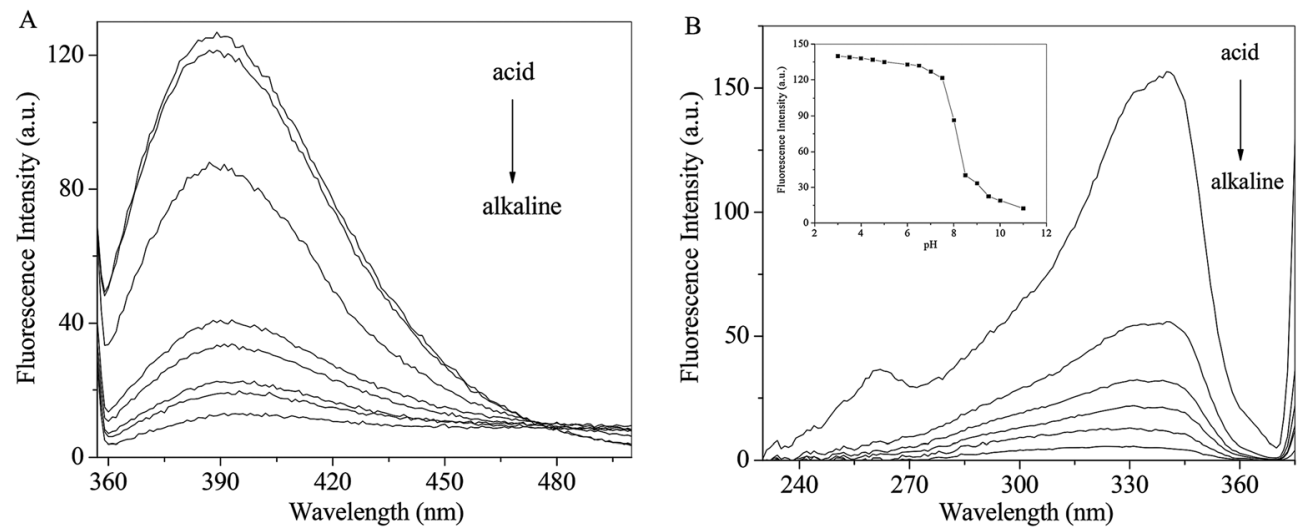

Fig. 5 Effect of $\mathrm{pH}$ on the emission (A) and excitation (B) spectra of $\mathrm{RH}$; insert: $\mathrm{pH}$ dependence of the fluorescence intensity of $\mathrm{RH}$ in methanol. The excitation and emission wavelengths were set at 340 and $390 \mathrm{~nm}$, respectively, $c(\mathrm{RH})=50.0 \mu \mathrm{M}$. 


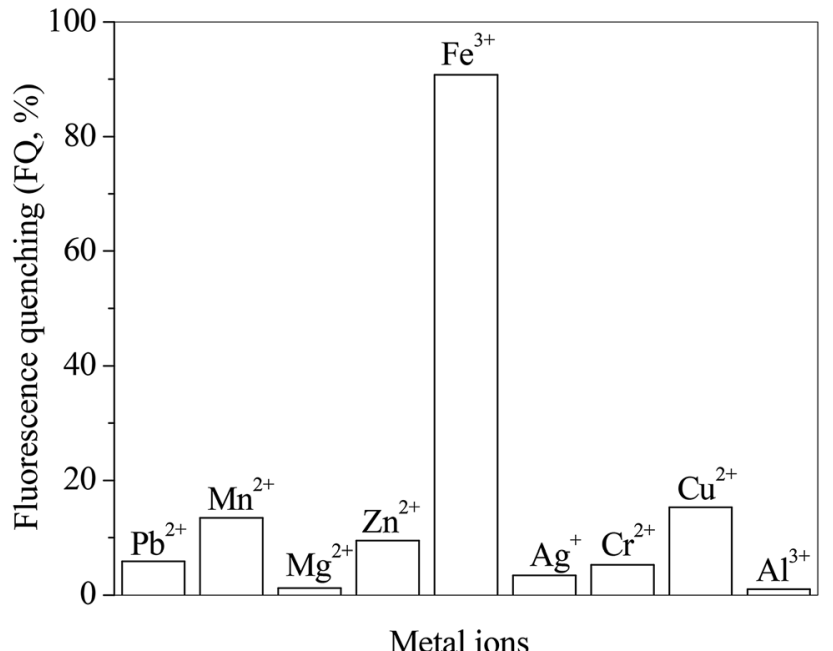

Fig. 6 Effect of different metal ions on the ratio of fluorescence quenching of $\mathrm{RH}$ in methanol. $c$ (metal ions) $=10.0 \mu \mathrm{M}, c(\mathrm{RH})=50.0$ $\mu$ M. $T=298 \mathrm{~K}$.

indicates that $\mathrm{Fe}^{3+}$ ion binds with $\mathrm{RH}$ non-covalently and a $\mathrm{RH}-$ $\mathrm{Fe}^{3+}$ complex forms.

Combining with the results of spectra and chromatography, we deduce that the binding of $\mathrm{Fe}^{3+}$ ion with $\mathrm{RH}$ occurs at the $\mathrm{C}$ 11 hydroxyl groups and the $\mathrm{O}$ atom of the methoxy group through hydrogen bond, and the decreasing electron donor ability facilitates the quenching (Fig. S3 $\uparrow$ ). And the double bond $\mathrm{C}=\mathrm{C}$ linking aromatic ring of $\mathrm{RH}$ molecule distorts more strictly by binding with $\mathrm{Fe}^{3+}$ ion.

\section{Binding parameters of interaction of $\mathrm{RH}$ with $\mathrm{Fe}^{3+}$}

Fluorescence quenching can occur by different mechanisms, usually classified as dynamic quenching and static quenching. In order to clarify the quenching mechanism, the Stern-Volmer equation was used to analyze the quenching data: ${ }^{27}$

$$
F_{0} / F=1+K_{\mathrm{SV}}[\text { iron }]
$$

where $F_{0}$ and $F$ are the fluorescence intensities in the absence and presence of quencher $\left(\mathrm{Fe}^{3+}\right.$ ion), respectively, $K_{\mathrm{SV}}$ is the Stern-Volmer quenching constant and [iron] is the concentration of $\mathrm{Fe}^{3+}$ ion. The values of $K_{\mathrm{SV}}$ could be measured from the slope by plotting $F_{0} / F$ against [iron] (Fig. S4A $\dagger$ ), and the corresponding results were listed in Table S1. $\dagger$ The binding constants and numbers of binding sites of interaction of $\mathrm{RH}$ with $\mathrm{Fe}^{3+}$ could be determined by the modified Stern-Volmer equation: ${ }^{21}$

$$
\lg \left[\left(F_{0}-F\right) / F\right]=\lg K+n \lg [\text { iron }]
$$

where $K$ is the binding constant, $n$ is the number of binding sites per $\mathrm{RH}$ for $\mathrm{Fe}^{3+}$ ion, and $F_{0}, F$ and [iron] have the same meanings as in eqn (12). The values of $K$ and $n$ are measured
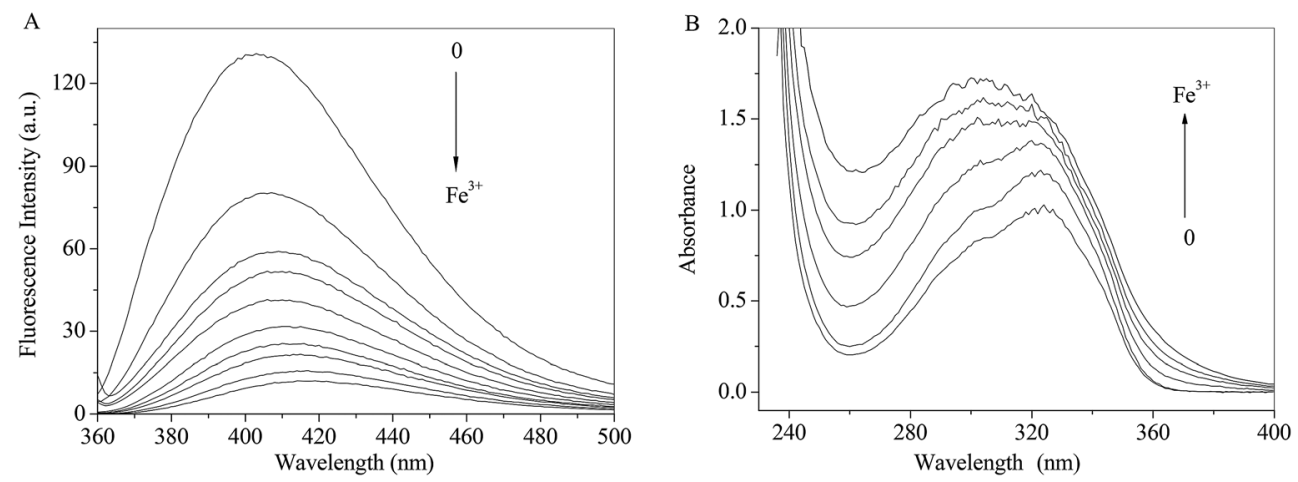

Fig. 7 Effect of $\mathrm{Fe}^{3+}$ ion on the fluorescence $(\mathrm{A})$ and absorption (B) spectra of $\mathrm{RH}$ in methanol. $c\left(\mathrm{Fe}^{3+}\right)=0-13.0 \mu \mathrm{M}, \mathrm{C}(\mathrm{RH})=50.0 \mu \mathrm{M} . T=298 \mathrm{~K}$.
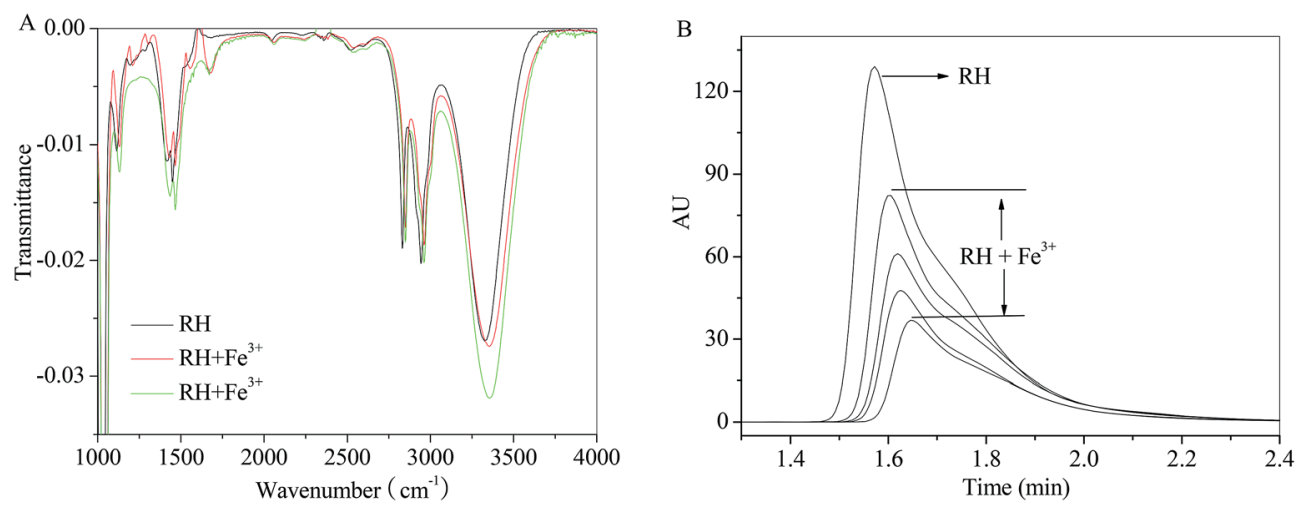

Fig. 8 Effect of Fe ${ }^{3+}$ ion on the FT-IR spectra and HPLC of RH in methanol. $c\left(\mathrm{Fe}^{3+}\right)=0-13.0 \mu \mathrm{M}, c(\mathrm{RH})=50.0 \mu \mathrm{M} . T=298 \mathrm{~K}$. 
from the intercept and slope by plotting $\lg \left[\left(F_{0}-F\right) / F\right]$ against $\lg$ [iron] (intercept $=\lg K$, slope $=n$ ) (Fig. S4 $\dagger$ ), and the corresponding results are listed in Table S1. $\dagger$ As shown in Table S1, $\dagger$ the value of $K_{\mathrm{Sv}}$ and $K$ is almost $10^{6} \mathrm{M}$, indicating that the quenching and binding effect is considerable, and $\mathrm{RH}$ can be candidate as a good source of new iron binding agents. The value of $n$ approximately is equaled to 1, indicating that there is one class binding site in $\mathrm{RH}$ for $\mathrm{Fe}^{3+}$. Because of the formation of the $\mathrm{RH}-\mathrm{Fe}^{3+}$ complex, the quenching of $\mathrm{RH}$ is initiated by static quenching.

\section{Conclusion}

In this work, solvent effect on the photophysical behavior of RH has been studied by absorption, fluorescence spectra and DFT/TDDFT calculations. The observed solvatochromic shift of spectra was used to calculate the ground and excited state pole moment of 2.23 and 3.67 D by Lippert-Suppan equation, respectively. Moreover, the increased Stokes shift in polar solvents suggests that RH is TICT feature, and correspond to $\pi \rightarrow \pi^{*}$ transitions. The photoluminescence behavior of $\mathrm{RH}$ is strongly modulated by the formation of intermolecular hydrogen bonds between $\mathrm{RH}$ and protic solvents. The excitation energy of the $\mathrm{S}_{1}$ state is calculated to be at 4.11 and $3.92 \mathrm{eV}$ for $\mathrm{RH}$ and the hydrogen bonded $\mathrm{RH}$, suggesting that the hydrogen bonding induces a decrease for the excitation energy of the $S_{1}$ state, and the hydrogen bond is strengthened in this excited state. Quantitative estimation of the relative contribution of several solvatochromic parameters indicates that the hydrogen-bond donor ability of the solvent is one of the factors governing the excited-state properties of $\mathrm{RH}$, which were verified by DFT/TDDFT calculations. The intermolecular hydrogen bonding occurred at the localized electron-acceptor oxygen at the glycoside bond. The effect of $\mathrm{pH}$ indicates that the fluorescence quenching of $\mathrm{RH}$ owing to the deprotonation of donor group and PET is facilitated in alkaline media. The $\mathrm{p} K_{\mathrm{a}}$ is 6.39 , which defines $\mathrm{RH}$ as a highly efficient "off-on" switcher. The interactions of RH with $\mathrm{Fe}^{3+}$ ion are studied by FT-IR and HPLC, and the binding parameter is calculated by the Stern-Volmer equation, and there is one class binding site of $\mathrm{RH}$ for $\mathrm{Fe}^{3+}$ ion. These experimental data would be available for clinical and analytical application of $\mathrm{RH}$ as an active drug and chromogenic reagent.

\section{Conflicts of interest}

There are no conflicts to declare.

\section{Acknowledgements}

This work was supported by the National Training Programs of Innovation and Entrepreneurship for Undergraduates (No. 201511080589).

\section{References}

1 G. Crisponia and M. Remelli, Coord. Chem. Rev., 2008, 252, 1225-1240.
2 N. Rupprich and H. Hildebrand, Arch. Biochem. Biophys., 1980, 200, 72-78.

3 Y. Y. Zhao, X. Y. Qin, S. P. Chen, Y. M. Zhang, R. C. Lin and W. J. Sun, J. Chem. Crystallogr., 2010, 10, 275-293.

4 Y. Y. Zhao, Q. Su, X. L. Cheng, X. J Tan, X. Bai and R. C. Lin, Bioanalysis, 2012, 6, 713-723.

5 Y. Y. Zhao, L. L. Wang, H. Miao, C. C. Ho, J. Li, X. L. Cheng, Y. M. Zhang, R. C. Lin and W. J. Sun, Chromatographia, 2011, 73, 599-603.

6 Y. Sun, Z. Ji, X. H. Liang, G. B. Li, S. Y. Yang, S. Wei, Y. Y. Zhao, X. Y. Hu and J. Fan, Spectrochim. Acta, Part A, 2012, 87, 171-178.

7 G. J. Zhao and K. L. Han, Acc. Chem. Res., 2012, 45, 404-413. 8 J. Jayabharathi, V. Thanikachalam, M. V. Perumal and N. Srinivasan, Spectrochim. Acta, Part A, 2011, 79, 236-244.

9 ADF 2010.01, SCM, Theoretical Chemistry, Vrije Universiteit, Amsterdam, The Netherlands, http://www.scm.com.

10 K. H. Birjkumar, N. D. Bryan and N. Kaltsoyannis, Dalton Trans., 2011, 40, 11248-11257.

11 G. J. Zhao and K. L. Han, ChemPhysChem, 2008, 9, 18421846.

12 P. A. Bouit, C. Villegas, J. L. Delgado, P. M. Viruela, R. P. Amérigo, E. Ortí and N. Martín, Org. Lett., 2011, 13, 604-607.

13 K. Saravanan, N. Srinivasan, V. Thanikachalam, J. Jayabharathi and J. Fluoresc, Chem. Phys. Lett., 2011, 21, 65-80.

14 A. Perrier, S. Aloïse, Z. Pawlowska, M. Sliwa, F. Maurel and J. Abe, Photoinduced intramolecular charge transfer process of betaine pyridinium: a theoretical spectroscopic study, Chem. Phys. Lett., 2011, 515, 42-48.

15 G. J. Zhao, J. Y. Liu, L. C. Zhou and K. L. Han, J. Phys. Chem. $B, 2007,111,8940-8945$.

16 B. Valeur, M. Nuno and B. Santos, Analytical Methods in Supramolecular Chemistry, Wiley-VCH, Weinheim, 2012.

17 A. Maciejewski1, E. Krystkowiak, J. Koput and K. Dobek, ChemPhysChem, 2011, 12, 1322-1332.

18 L. Cisse, A. Djande, M. C. Chichi, F. Delatre, A. Saba, A. Tine and J. J. Aaron, Spectrochim. Acta, Part A, 2011, 79, 428-436.

19 Z. Pawlowska, A. Lietard, S. Aloïse, M. Sliwa, A. Idrissi, O. Poizat, G. Buntinx, S. Delbaere, A. Perrier, F. Maurel, P. Jacques and J. Abe, Phys. Chem. Chem. Phys., 2011, 13, 13185-13195.

20 A. Perrier, S. Aloïse, Z. Pawlowska, M. Sliwa, F. Maurel and J. Abe, Chem. Phys. Lett., 2011, 515, 42-48.

21 K. M. Al-Ahmary, M. M. Habeeb and E. A. Al-Solmy, J. Mol. Liq., 2011, 162, 129-134.

22 G. J. Zhao and K. L. Han, J. Chem. Phys., 2007, 127, 024306. 23 G. J. Zhao and K. L. Han, Biophys. J., 2008, 94, 38-46.

24 D. P. Yang, Y. G. Yang and Y. F. Liu, Comput. Theor. Chem., 2012, 997, 42-48.

25 N. I. Georgiev, V. B. Bojinov and P. S. Nikolov, Dyes Pigm., 2011, 88, 350-357.

26 J. M. Chovelon and I. Grabchev, Spectrochim. Acta, Part A, 2007, 67, 87-91.

27 Y. J. Hu, Y. Liu, R. M. Zhao, J. X. Dong and S. S. Qu, J. Photochem. Photobiol., A, 2006, 179, 324-329. 